\title{
SCUAD and chronic rhinosinusitis. Reinforcing hypothesis driven research in difficult cases*
}

\author{
Emmanuel P. Prokopakis ${ }^{1}$, loannis M. Vlastos' ${ }^{1}$, Berrylin J. Ferguson², \\ Glenis Scadding ${ }^{3}$, Hideyuki Kawauchi ${ }^{4}$, Christos Georgalas ${ }^{5}$, \\ Nikolaos Papadopoulos ${ }^{6}$, Peter W. Helling $5^{5,7}$ \\ 1 Department of Otorhinolaryngology, University of Crete School of Medicine, Heraklio, Crete, Greece \\ 2 Department of Otolaryngology, University of Pittsburgh School of Medicine, Pittsburgh, USA \\ ${ }^{3}$ Department of Allergy and Medical Rhinology, Royal National TNE Hospital, London WC1X8DA, United Kingdom \\ ${ }^{4}$ Department of Otorhinolaryngology, Shimane University, Faculty of Medicine, Izumo, Japan \\ ${ }^{5}$ Department of Otorhinolaryngology, Academic Medical Centre, Amsterdam \\ ${ }^{6}$ Department of Pedo-Allergiollogy, University of Athens, Greece \\ ${ }^{7}$ Department of Otorhinolaryngology, University Hospitals Leuven, Leuven, Belgium
}

Rhinology 52: 3-8, 2014

DOl:10.4193/Rhino13.049

*Received for publication:

April 29, 2013

Accepted: September 12, 2013

\begin{abstract}
Background: Our objective is to present recent research findings on recalcitrant chronic rhinosinusitis (CRS) in relation to "Severe Chronic Upper Airway Disease" (SCUAD).
\end{abstract}

Methodology: Literature review using Medline and Embase databases (search terms 'chronic rhinosinusitis", "chronic sinusitis" or "Severe Chronic Upper Airway Disease") limited to articles published in the English language.

Results: Complex pathophysiological mechanisms characterize various forms of chronic rhinitis and rhinosinusitis (CRS), where inflammation persists in spite of adequate medical treatment. In these cases, a multifactorial etiology often underlies the development of sino-nasal inflammation. The interaction between chronic upper and lower airway inflammation via neurogenic and systemic pathways may complicate the therapy of these patients, and lead to insufficient symptom control.

Conclusion: The recently introduced definition of "Severe Chronic Upper Airway Disease" (SCUAD) increases awareness of those patients with persistent inflammation and symptoms despite guideline-driven pharmacologic treatment. The concept of SCUAD may prove helpful in directing research towards clarifying the definition, diagnosis and pathophysiology of rhinitis and rhinosinusitis, their limits and overlap. In this review, a hypothesis on SCUAD immunopathology is also presented.

Key words: chronic rhinosinusitis, severe chronic upper airways disease, definitions, pathophysiology

\section{Introduction}

Severe Chronic Upper Airway Disease (SCUAD) is a term recently introduced by Bousquet et al. ${ }^{(1)}$ defining those patients with allergic (AR), non-allergic (NAR), and occupational rhinitis (OR) and chronic rhinosinusitis (CRS) with and without nasal polyposis, whose symptoms are inadequately controlled despite pharmacological treatment following international validated guidelines, such as ARIA ${ }^{(2,3)}$ and EPOS2012 ${ }^{(4)}$. Chronic inflammatory disorders of the upper airways are extremely prevalent and have a major impact on public health and the socioeconomic state ${ }^{(2,3)}$. It is estimated that up to one third of rhinitis and rhinosinusitis patients present with persistent inflammation and/or symptoms despite adequate treatment ${ }^{(3,4)}$. In other words, a great number of these patients fail to respond to treatment options suggested by guidelines such as antibiotics, steroids or surgery. Moreover, these disorders commonly complicate diagnosis and management of diseases of the lower airways. The group of SCUAD patients is a subgroup of patients that challenge the currently 
available treatment schemes and stress the importance of research in the field of difficult to treat rhinitis and rhinosinsusitis patients.

Although rhinitis is not a life-threatening disease, patients with this condition and especially those with SCUAD have a severely impaired quality of life ${ }^{(5)}$. Moreover, patients with CRS have more bodily pain and worse social functioning than patients with chronic obstructive pulmonary disease (COPD), congestive heart failure, or back pain ${ }^{(6)}$. CRS represents a significant health problem, resulting in frequent surgical procedures and a large financial burden on society ${ }^{(4)}$. Despite recent clinical and basic research efforts, the pathophysiology of the disease is only beginning to be understood. Current research has reinforced the belief that CRS is a multifactorial disease process involving genetic, environmental, occupational, anatomic, iatrogenic and immunological factors ${ }^{(7)}$. Based on the concept of "united airways", new diagnostic and therapeutic approaches are required to delineate whether intermittent and persistent inflammatory disorders of the upper airways represent a common disease with strong relationships with asthma and other diseases, or are distinct entities that interact with each other or are simply being confused because of common symptoms (Figure 1). The scope of this review is to highlight the arguments and evidence on each of the aforementioned hypotheses and how these relate to SCUAD, to reinforce clinical and basic research towards a better delineation of these issues.

\section{SCUAD}

Definition issues

Attempts have been made to define rhinosinusitis in terms of pathophysiology, microbiology, radiology, severity and duration of symptoms ${ }^{(8-10)}$. However, the predictive value of the symptom-based definition of CRS, commonly used in epidemiological studies, falls behind that which includes objective tests ${ }^{(11)}$. The EP3OS document ${ }^{(4)}$ offers definitions for clinical and research use, as well as for epidemiological studies. Despite the initial results showing that symptom-based CRS is significantly associated with positive endoscopy and with self-reported doctordiagnosed CRS ${ }^{(12)}$, further adjustments are needed because they have a moderate reliability over time ${ }^{(12)}$. Moreover, a more recent study ${ }^{(13)}$ questioned whether these definitions evaluate the same disease due to their moderate agreement. In addition, signs and symptoms of allergic and non allergic rhinitis overlap. These entities are routinely differentiated by the presence or absence of an overt lgE- mediated pathogenic mechanism, however local lgE may be relevant ${ }^{(14)}$.

\section{Related etiological factors}

Despite previous studies suggesting that there is an increased incidence of acute and chronic rhinosinusitis in patients with allergic rhinitis ${ }^{(15-17)}$ more recent evidence suggests that allergic rhinitis is an associated condition rather that a trigger for CRS ${ }^{(18)}$. Several mechanisms have been studied regarding the pathophysiology of rhinosinusitis in association to allergic and non-allergic rhinitis, such as the role of mucociliary clearance. However, the role of sinonasal cilia, their response to environmental stimuli and their function under several conditions remains unclear ${ }^{(19,20)}$. For example, cystic fibrosis (CF) and primary ciliary dyskinisia (PCD) both cause disturbances of mucociliary clearance and are commonly implicated in difficult-to-treat CRS cases. Nevertheless, CF, unlike PCD ${ }^{(21)}$, often presents as CRS with nasal polyps and is associated with infection and biofilms, indicating the existence of complex molecular and biochemical mechanisms that are incompletely understood. The current development of nasal and lung biomarkers, such as nitric oxide (22), for the differential diagnosis of those diseases signifies the importance of further basic and translational research studies. The reverse interaction, namely the impact of microbial colonization and/or environmental agents on allergic and non-allergic rhinitis, remains incompletely understood. Microbial infections and bacterial products, such as Staphylococcus aureus enterotoxin $B$ have been implicated in inflammatory responses ${ }^{(23)}$, and have been shown to facilitate allergic sensitization ${ }^{(24)}$. However, the complexity of the issue can be seen by the studies on the hygiene hypothesis ${ }^{(25)}$. Bacterial, viral or other infectious factors modulate T-cell responses differently and time of exposure can modulate the outcome. Several molecules and mechanisms are being investigated. For example, the expression of innate immune markers, such as Toll-like Receptor-9 (TLR9), has been shown to be upregulated in response to repeated microbial insults ${ }^{(26)}$. TLRs are implicated in the hygiene hypothesis and several experimental (with TLR agonists), genetic (mostly in relations to TLR polymorphisms) and epigenetic data are now available. However, information gathered on the issue is insufficient for robust verification of the vicious cycle theory reported in Figure 1. In other words, unless the complexity of the molecular interactions between assaulting factors and immunological host responses as well as their biochemical consequences is resolved, the hypothesis that Severe Chronic Upper Airway Diseases are caused by a dysfunction of the feedback mechanism in some patients, cannot be supported.

\section{A single disease?}

Multiple pathophysiological pathways involving innate and adaptive immune responses, specific cytokine profiles, molecular mechanisms at the level of epithelial cells, surface, microenvironment or intracellular matrix have been found in common in allergic and non-allergic rhinitis and CRS. A hypothesis that can be generated is that the minority of patients with allergic rhinitis, non-allergic rhinitis and CRS, who have symptoms refractory to standard medical therapy and sinus surgery procedures, may 


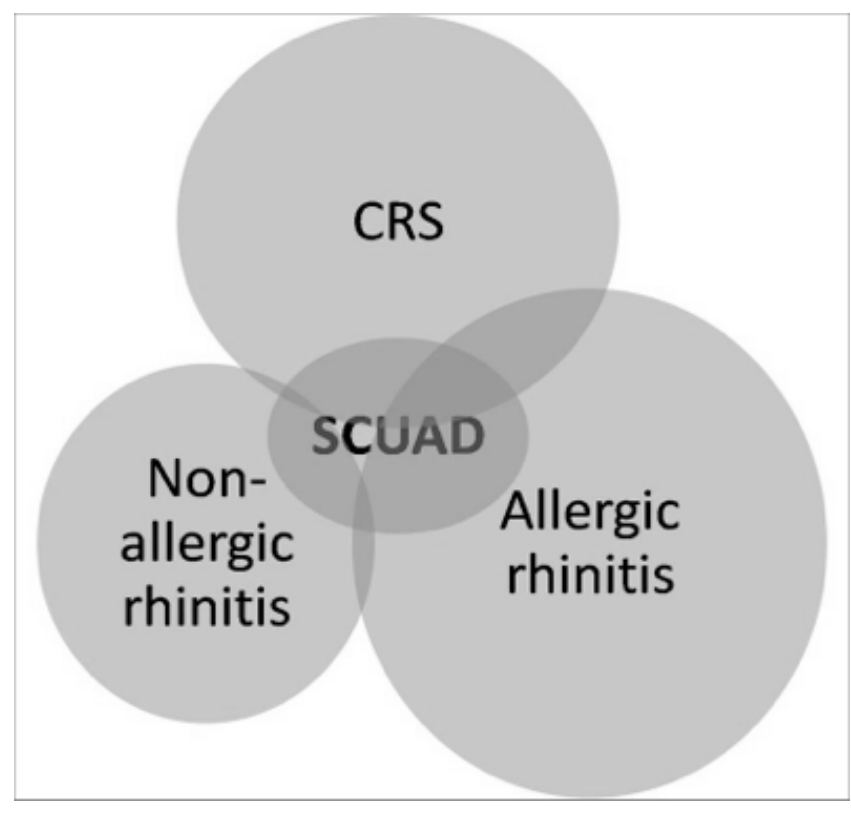

Figure 1. A vicious cycle, a common disease or distinct entities with common symptoms?

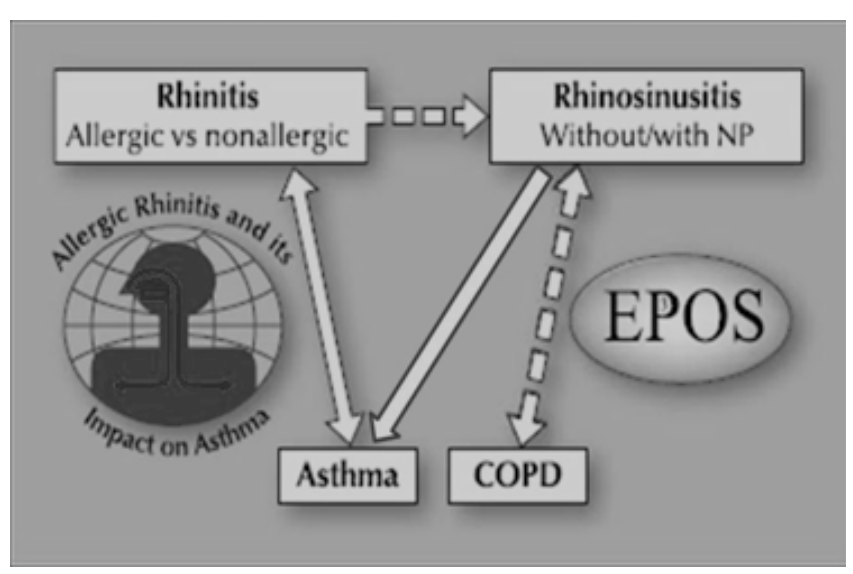

Figure 2. Scheme depicting the interaction between rhinitis and rhinosinusitis in relation to bronchial pathology. The two state-of-the art documents (ARIA [Allergic Rhinitis and its Impact on Asthma] and EP3OS [European Position Paper on Rhinosinusitis and Nasal Polyps, 2007]) are listed, as they contain overviews of current knowledge on nasobronchial interaction. COPD: chronic obstructive pulmonary disease; NP: nasal polyps (Illustration by P.W. Hellings and E.P. Prokopakis Global Airway Disease Beyond Allergy. Curr Allergy Asthma Rep. 2010; 10: 143-149, used with permission). have a common underlying pathophysiological condition that presents as Severe Chronic Upper Airway Disease.

The "united airways concept" (Figure 2) implies that besides the anatomical continuity of the upper and lower airways, inflammation in one part of the airway influences the homeostasis of the other ${ }^{(27)}$. The mechanisms underlying this interaction have been studied primarily in allergic disease, showing systemic immune activation, induction of inflammation at a distance, and a negative impact of nasal inflammation on bronchial homeostasis ${ }^{(27)}$. In addition to allergy, the concept of global airway disease has been demonstrated among others in patients with nonallergic asthma and in those with COPD who present with sinonasal symptoms and inflammatory markers in nasal lavages ${ }^{(28)}$. To further expand the idea of a broader causal mechanism the term allergic rhinosinusitis has been adopted by some authors (29). Several hypotheses have been vigorously debated during the past decade with the majority of studies implicating fungi, biofilms and superantigens in the pathogenesis of CRS. Several of them have been influenced by analogous studies on asthma. A T-cell-driven, non-lgE-mediated hypersensitivity response that culminates in the attraction and specific targeting of eosinophils against colonized fungi in the nasal lumen of CRS patients, with subsequent degranulation and mucosal damage, has been proposed as a possible mechanism of CRS (30). This hypothesis, the so-called fungal hypothesis, which strongly relates allergic rhinitis, non-allergic rhinitis and CRS, suggests that high levels of Alternaria trigger the accumulation of peripheral blood mononuclear cells and eosinophils. Still, evidence is lacking if this is a disease-specific response or whether Alternaria has any relevance to the establishment of $C R{ }^{(31)}$. Moreover, new culture techniques revealed that the percentage of positive fungi cultures was the same in normal controls and in CRS patients (32). Finally, later clinical studies examining the effect of topical antifungal agents were disappointing ${ }^{(33,34)}$.

Immune responses are being implicated also in the superantigen (SAg) hypothesis, which incorporates biofilm formation and toxin secretion mainly by S. aureus. The biofilm has been described as an aggregation of microorganisms embedded in a protective self-produced polysaccharide matrix. According to the superantigen hypothesis, S. aureus protected by biofilms or sequestered within epithelial cells secrete toxins that result in a generalized stimulation of $\mathrm{T}$ cells, cytokine release, and a local polyclonal IgE response, all of which stimulate eosinophil recruitment and the clinical and histopathological changes associated with polypoid CRS ${ }^{(31)}$. Although about half of CRS with nasal polyposis patients show evidence of Streptococcal Ag responses, multiple pathophysiological pathways likely exist for the development of CRS. This is an evolving field of knowledge and even the role of biofilms is being disputed lately by researchers who believe that the biofilm in the nose and paranasal sinuses is "nothing else but regular respiratory mucosal blanket, a part of the mucociliary system itself, and containing variable number of bacteria" (35). In terms of therapeutic implications, further research is required to clarify whether prophylactic anti- 


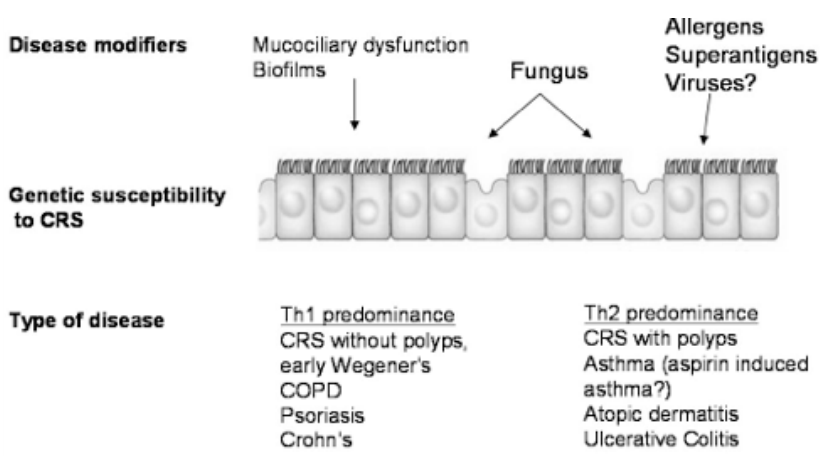

Figure 3. Heterogeneity of CRS.

bacterial treatment should differ between primary and revision sinus surgery.

Moreover, accumulated data on the pathophysiology of CRS have led some researchers to propose SAgs as disease modifiers (Figure 3). The depicted immune barrier hypothesis acknowledges CRS as a member of a family of chronic inflammatory disorders that occur at sites of interface with the outside world. Antigen passage and processing across the nasal epithelium is promoted by defects of the mechanical and innate immune protective barrier that leads to the generation of the chronic inflammatory infiltrate observed in CRS ${ }^{(31)}$. As proposed by Kern et al. (31), "variations in the expression of genes that govern critical host epithelial pathways may increase the susceptibility to CRS. Environmental factors, rather than discrete etiological agents, can be seen as disease modifiers that skew the clinical presentation in an individual patient." However, this has been criticized as being over simplistic ${ }^{(9)}$ since it may underestimate the possible role of T regulatory cells (Treg), dendritic cells or other factors such as Th17 cells. Moreover, possible abnormal innate immune responses of sinonasal epithelial cells are being ignored, such as diminished activity of TLRs found in recalcitrant CRS with nasal polyps ${ }^{(37,38)}$.

\section{SCUAD immunopathology}

The not well delineated pathophysiological mechanism of recalcitrant CRS has lead as to propose a symptom-based treatment algorithm ${ }^{(39)}$. However, optimal treatment cannot be based on partial symptoms control. The question whether SCUAD represents a single disease is a result of a disrupted feedback mechanism in patients with CRS, allergic or non allergic rhinitis, or is just the clinical presentation of different entities reflects our lack of understanding of the pathophysiology at cellular and molecular levels.

Clearly severe chronic upper airway disease is a chronic and deliberating disease, in which "abnormal" innate and adaptive immune responses can be encountered. Exogenous factors, such as microbes in combination with intrinsic factors, such as common primary immunodeficiency disease, primary ciliary dyskinesia or cystic fibrosis can contribute to a constant Th1 activation, that favors tissue remodeling and fibrosis seen in patients with CRS without nasal polyps. Likewise, environmental pollutants and allergens in "susceptible individuals" elicit a Th2 response. The Th2-biased mucosal inflammation, in turn, favours the programming of $\mathrm{M} 2$ macrophages within the polyp tissue in CRS with nasal polyps ${ }^{\left({ }^{(0)}\right)}$ and may contribute to polyps formation. This process can be further violated by a deficiency in $T$ regulatory cells or deregulation in cytokines production, like IL-2 or IL-5, attributed to several genetic and epigenetic factors. Overwhelming data have also been published on the epithelial expression of cytokines that regulate Th2 cytokine responses (i.e., thymic stromal lymphopoietin, IL-25, and IL-33). To further complicate the issue, multiple pathways are involved in the interaction between T cells and other immune cells, like eosinophiles, dentritic cells and baseophils, seen in rhinitis patients (i.e. CRTH2 antagonism is currently being studied as a new therapeutic approach for allergic patients ${ }^{(41,42)}$ ). An example of the complex inflammatory response in allergic rhinitis is reviewed in (43). In SCUAD, however, chronic, constant and serious inflammation leads to alternation of expression and concentration of cytokines, ligands, receptors, etc, that obscure the primary deficiency.

In contrary to SCUAD patients, the majority of CRS, allergic and non-allergic rhinitis cases, that are relatively easily treated, have known extrinsic and intrinsic factors and current therapeutic regimens (i.e. antibiotics, immunotherapy) "alleviate" immune responses. After the exclusion of incorrect diagnoses and specific causes of difficult to treat CRS cases (reviewed in ${ }^{(44)}$ and ${ }^{(45)}$ ), such as odontogenic inflammation, a significant percentage of patients is reported as having SCUAD ${ }^{(44)}$. It is of interest that an alternation of cytokines production by epithelial and immune cells, as those reported very briefly in the above paragraphs, can cause a failure of the regulation of Th1 and Th2 responses. Our hypothesis, depicted in Figure 4, is that apart from extrinsic and intrinsic factors that can contribute to a constant inflammation characterized either by Th1 or Th2 predominance, defective pathways involved in Th1 vs Th2 response regulation may be the single or just a simple cause of SCUAD.

\section{Unmet needs and future research directions}

CRS refractory to standard medical and surgical treatments is a common and debilitating condition. Disease development remains incompletely understood with multiple pathophysiological pathways being proposed, but still requiring investigation. To delineate factors that contribute to severe CRS, epidemiological, clinical and molecular studies are required.

The real prevalence and severity of CRS/SCUAD, as well as its effect on social/professional life and costs should be determined by well conducted epidemiological studies. First of all, an 


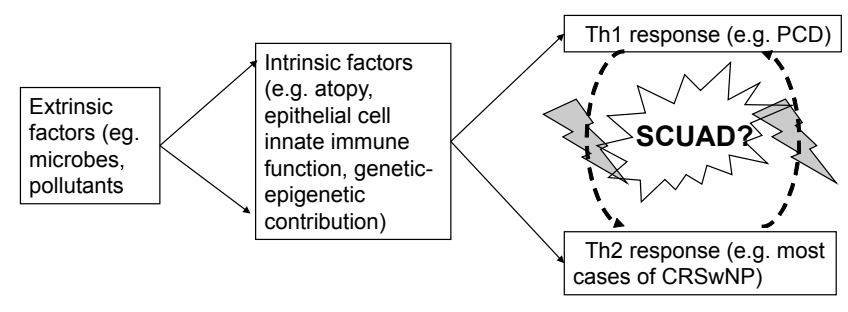

Figure 4. SCUAD can be attributed to a single primary deficiency in Th1 vs Th2 responses regulation or maybe a multifactorial disease with multiple defective pathways leading to common CRS symptoms.

explicit definition of control of disease is required. The concept of control of disease has only recently been introduced in the field of AR and CRS (44). As it has already been mentioned in the section of the SCUAD pathophysiology, in case of poor control of symptoms despite guideline-directed pharmacotherapy, one needs to consider the presence of SCUAD but also treatmentrelated, diagnosis-related and/or patient-related factors ${ }^{(44)}$. Furthermore, contributing factors such as exposure to environmental or occupational agents, underlying gastroesophageal reflux, and/or infection or colonization with micro-organisms need better understanding ${ }^{(28)}$. Fungal extracts and bacterial enterotoxins were linked to the etiology of nasal polyposis. Nevertheless, only few studies have examined viral contribution to pathogenesis of the mucosal inflammation, although viruses are widely considered as a triggering factor, especially in aspirinsensitive rhinosinusitis ${ }^{(46,47)}$. Atypical bacteria, such as Mycoplasma and Chlamydia may also hold an unidentified role (27). Recent mechanistic studies have revealed a complex bidirectional communication between sinonasal epithelial cells and the adaptive immune system ${ }^{(36)}$. In addition, the cellular sources as well as the mechanisms of systemic release of proinflamma- tory mediators (eg, IL-5) are still unknown ${ }^{(28)}$. To design novel treatments, a better understanding of disease mechanisms is needed.

Researchers work towards identifying key genetic polymorphisms and epigenetic variations and how they promote disease susceptibility in the individual patient ${ }^{(31,48)}$. A better understanding of the local features of the chronically inflamed epithelium will be essential for the developing of new diagnostic and therapeutic tools. It is intriguing that roots of modern topical treatments can be found in early knowledge of rhinological disease, as presented in texts of antiquity ${ }^{(49)}$. Targeting therapies to intervene in the particular defective pathway(s) present may be preferable to broad suppression of inflammation or extensive efforts to reduce microbial colonization with antibiotics ${ }^{(31)}$ and are expected to be a breakthrough of current evolution of molecular biology.

SCUAD is not a lethal disease, thus new regimens that are related to immune function and especially to T cells interactions, are expected to be utilized in other more serious diseases first, as it has already done with IgE, IL-5 and CRTH2 antagonists. Nevertheless, the concept of SCUAD will allow clinical and molecular research efforts to be directed towards difficult and treatmentunresponsive CRS.

\section{Authorship contribution}

EPP and IMV contributed in conception and design of the study, acquisition, analysis and interpretation of data and drafting of the manuscript, whereas BJF, GS, HK, CG, NP and PWH participated in analysis and interpretation of data and revised the article.

\section{Conflict of interest}

There was no conflict of interest in this study.

\section{References}

1. Bousquet J, Bachert C, Canonica GW, et al. Unmet needs in severe chronic upper airway disease (SCUAD), J Allergy Clin Immunol. 2009; 124: 428-433.

2. Bousquet J, Van Cauwenberge $\mathrm{P}$, Khaltaev N. Allergic rhinitis and its impact on asthma. J Allergy Clin Immunol. 2001; 108: S147-334.

3. Bousquet J, Khaltaev N, Cruz AA, et al. Allergic Rhinitis and its Impact on Asthma (ARIA) 2008 update (in collaboration with theWorld Health Organization, GA(2)LEN and AllerGen). Allergy 2008; 63 (suppl 86): 8-160.

4. Fokkens WJ, Lund VJ, Mullol J, et al. EPOS 2012: European position paper on rhinosinusitis and nasal polyps 2012. Rhinology supplement 2012; 23: 1-298.

5. Greiner AN, Hellings PW, Rotiroti G, Scadding GK. Allergic rhinitis. Lancet. 2011;
17: 378: 2112-2122.

6. Gliklich RE, Metson R. The health impact of chronic sinusitis in patients seeking otolaryngologic care. Otolaryngol Head Neck Surg. 1995; 113: 104-109.

7. Ramakrishnan VR, Kennedy DW. Advances in the Surgical Management of Chronic Sinusitis and Nasal Polyps. Curr Allergy Asthma. 2011; 11: 220-229.

8. Shapiro GG, Rachelefsky GS. Introduction and definition of sinusitis. J Allergy Clin Immunol. 1992; 90: 417-418.

9. Williams JJr, Simel DL. Does this patient have sinusitis? Diagnosing acute sinusitis by history and physical examination. JAMA. 1993; 270: 1242-1246.

10. Lund VJ. Infectious rhinosinusitis in adults: classification, etiology and management. ENT J. 1997; 76: 1-22.

11. Tahamiler R, Canakcioglu S, Ogreden S,
Acioglu E. The accuracy of symptom-based definition of CRS. Allergy. 2007; 62: 10291032.

12. Tomassen P, Newson RB, Hoffmans R, et al. Reliability of EP3OS symptom criteria and nasal endoscopy in the assessment of chronic rhinosinusitis--a GA ${ }^{2}$ LEN study. Allergy. 2011; 66: 556-561.

13. Lange B, Thilsing T, Baelum J, Holst R, Kjeldsen A. Diagnosing chronic rhinosinusitis: comparing questionnaire-based and clinical-based diagnosis. Rhinology. 2013; 51: 128-136.

14. Bousquet J, Bieber T, Fokkens WJ, et al. Important questions in allergy: novel research areas. Allergy. 2008; 63: 143-147.

15. Derebery MJ. Otolaryngic allergy. Otolaryngol Clin North Am. 1993; 26: 593611.

16. Berrettini S, Carabelli A, Sellari-Franceschini 
S, et al. Perennial allergic rhinitis and chronic sinusitis: Correlation with rhinologic risk factors. Allergy. 1999; 54: 242-248.

17. Hoover GE, Newman LJ, Platts-Mills TA, Phillips CD, Gross CW, Wheatley LM. Chronic sinusitis: Risk factors for extensive disease. Allergy Clin Immunol. 1997; 100: 185-191.

18. Hens G, Vanaudenaerde BM, Bullens DM, et al. Sinonasal pathology in nonallergic asthma and COPD: 'united airway disease' beyond the scope of allergy. Allergy. 2008; 63: 261-267.

19. Vlastos I, Athanasopoulos I, Mastronikolis N Impaired mucociliary clearance in allergic rhinitis patients is related to a predisposition to rhinosinusitis. Ear Nose Throat 2009; 88: E17-19.

20. Cohen NA, Sinonasal mucociliary clearance in health and disease. Ann Otol Rhino Laryngol. Suppl 2006; 196: 20-26.

21. Rollin M, Seymour K, Hariri M, Harcourt J. Rhinosinusitis, symptomatology \& absence of polyposis in children with primary ciliary dyskinesia. Rhinology. 2009; 47: 75-78.

22. Scadding G, Scadding GK. Update on the use of nitric oxide as a noninvasive measure of airways inflammation. Rhinology. 2009; 47: 115-120.

23. Bachert C, Zhang N, van Zele T, Gevaert $P$, Patou J, van Cauwenberge $P$. Staphylococcus aureus enterotoxins as immune stimulants in chronic rhinosinusitis. J Clin Allergy Immunol. 2007; 20: 163175.

24. Huvenne W, Callebaut I, Plantinga M, et al. Staphylococcus aureus enterotoxin B facilitates allergic sensitization in experimental asthma. Clin Exp Allergy. 2010; 40: 1079-1090

25. Prokopakis E, Vardouniotis A, Kawauchi H, et al. The pathophysiology of the hygiene hypothesis. Int J Pediatr Otorhinolaryngol. 2013; 77: 1065-1071.

26. Melvin TA, Lane AP, Nguyen MT, Lin SY Allergic rhinitis patients with recurrent acute sinusitis have increased sinonasal epithelial cell TLR9 expression. Otolaryngol Head Neck Surg. 2010; 142: 659-664.

27. Hellings PW, Prokopakis EP. Global Airway Disease Beyond Allergy. Curr Allergy Asthma Rep. 2010; 10: 143-149.

28. Hens G, Vanaudenaerde BM, Bullens DM, et al. Sinonasal pathology in nonallergic asthma and COPD: 'united airway disease' beyond the scope of allergy. Allergy 2008; 63: 261-267.
29. Trevino RJ, Gordon BR, Allergic rhinosinusitis: The total rhinologic disease. Ear Nose Throat J. 1993; 72: 121-125.

30. Inoue $Y$, Matsuwaki $Y$, Shin $\mathrm{SH}$, et al. Nonpathogenic, environmental fung induce activation and degranulation of human eosinophils. J Immunol. 2005; 175 5439-5447.

31. Kern RC, Conley DB, Walsh W, Chandra R, Kato A, Tripathi-Peters A, et al. Perspectives on the etiology of CRS: An immune barrier hypothesis. Am J Rhinol. 2008; 22: 549-559.

32. Braun $H$, Stammberger $H$, Buzina W Freudenschuss K, Lackner A, Beham A. Incidence and detection of fungi and eosinophilic granulocytes in CRS. LaryngoRhino- Otologie. 2003; 82: 330-340.

33. Ebbens FA, Scadding GK, Badia $L$, et al Amphotericin B nasal lavages: Not a solution for patients with CRS. J Allergy Clin Immunol. 2006; 118: 1149-1156.

34. Weschta M, Rimek D, Formanek M, Polzehl D, Podbielski A, Riechelmann H. Topical antifungal treatment of CRS with nasal polyps: a randomized, double-blind clinical trial, J Allergy Clin Immunol. 2004; 113: 1122-1128.

35. Mladina R, Skitarelic N. Biofilm - The other name for the regular mucosal blanket. Medical Hypotheses. 2010; 75: 391-392.

36. Lee S, Lane AP. Chronic rhinosinusitis as a multifactorial inflammatory disorder. Curr Infect Dis Rep. 2011; 13: 159-168.

37. Wang J, Matsukura S, Watanabe $S$, et al. Involvement of Toll-like receptors in the immune response of nasal polyp epithelial cells. Clin Immunol. 2007; 124: 345-352.

38. Vandermeer J, Sha Q, Lane AP, Schleimer RP. Innate immunity of the sinonasal cavity: expression of messenger RNA for complement cascade components and toll-like receptors. Arch Otolaryngol Head Neck Surg. 2004; 130: 1374-1380.

39. Prokopakis E, Vlastos I, Pant H, Ferguson BJ. Chronic rhinosinusitis: observation or treatment. Curr Opin Allergy Clin Immunol. 2013; 13: 31-36C

40. Bachert C, Zhang N. Chronic rhinosinusitis and asthma: novel understanding of the role of IgE 'above atopy'. J Int Med 2012; 272: 133-143

41. Fajt ML, Gelhaus SL, Freeman B, et al. Prostaglandin D2 pathway upregulation: Relation to asthma severity, control, and $\mathrm{TH} 2$ inflammation. Allergy Clin Immunol. 2013: 131:1504-1512.
42. Horak F, Zieglmayer P, Zieglmayer R, et al. The CRTH2 antagonist OC000459 reduces nasal and ocular symptoms in allergic subjects exposed to grass pollen, a randomised, placebo-controlled, double-blind trial. Allergy. 2012; 67: 1572-1579.

43. Pawankar R, Mori S, Ozu C, Kimura S Overview on the pathomechanisms of allergic rhinitis. Asia Pac Allergy. 2011; 1: 157167

44. Hellings PW, Fokkens WJ, Akdis C, et al. Uncontrolled allergic rhinitis and chronic rhinosinusitis: where do we stand today? Allergy. 2013; 68: 1-7.

45. Woodbury K, Ferguson BJ. Recalcitrant chronic rhinosinusitis: investigation and management. Curr Opin Otolaryngol Head Neck Surg. 2011; 19: 1-5.

46. Szczeklik A, Aspirin-induced asthma as a viral disease. Clin Allergy. 1988; 18: 15-20.

47. Papadopoulos NG, Xepapadaki P, Mallia P et al, Mechanisms of virus-induced asthma exacerbations: state-of-the-art. A GA2LEN and InterAirways document. Allergy. 2007; 62: 457-470.

48. Bachert C, Van Bruaene N, Toskala E, et al. Important research questions in allergy and related diseases: 3-CRS and nasal polyposis - a GA2LEN study. Allergy. 2009; 64: 520533

49. Prokopakis EP, Hellings PW, Velegrakis GA Kawauchi H. From ancient Greek medicine to EP ${ }^{3}$ OS. Rhinology. 2010; 48: 265-272.

Emmanuel P. Prokopakis, MD, Ass. Professor

Department of Otorhinolaryngology University of Crete School of Medi-

cine

Voutes

71110, Heraklion

Crete

Greece

Tel: +3-2810392347

E-mail: eprokopakis@gmail.com 